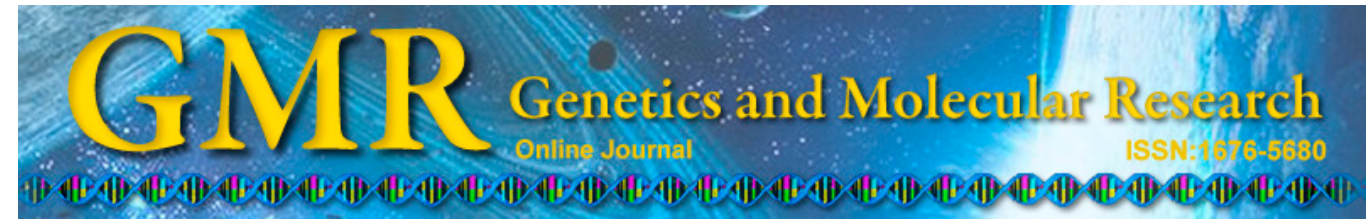

\title{
Administration of low-dose cyclosporine alone for the treatment of elderly patients with membranous nephropathy
}

\author{
M.X. Li, Y.W. Yu, Z.Y. Zhang, H.D. Zhao and F.L. Xiao \\ Department of Nephrology, The Navy General Hospital, Beijing, China \\ Corresponding author: M.X. Li \\ E-mail: mingxulicn@163.com
}

Genet. Mol. Res. 14 (1): 2665-2673 (2015)

Received May 27, 2014

Accepted November 3, 2014

Published March 30, 2015

DOI http://dx.doi.org/10.4238/2015.March.30.27

\begin{abstract}
This study aimed to investigate the effects of administration of low-dose cyclosporine A (CsA) alone and the combination of low-dose CsA and a low-dose hormone for the treatment of elderly patients with membranous nephropathy. We divided 27 patients into two groups as follows: low-dose CsA group (group A) and the group receiving a combination of a low-dose hormone and low-dose CsA (group B). The treatment and follow-up times were $\geq 6$ months. We observed no difference in gender, age, serum creatinine levels, estimated glomerular filtration rate (eGFR), and 24-h urinary protein levels between the two groups before treatment; in addition, the rates of complete and partial remission were not different 6 months after treatment. The rate of complications in group B was higher than that in group A ( $84.6 v s 35.7 \%$, respectively; $\mathrm{t}=0.018)$. While the pretreatment eGFR of patients who achieved remission was significantly higher than that of patients who did not achieve remission, the 24-h urinary protein levels and incidence of hypertension were significantly lower than those of patients who did not achieve remission $(t=0.042,0.035$ and 0.043 , respectively). The efficacy of administration of low-dose CsA alone and in combination with a low-dose hormone was similar; the
\end{abstract}


efficacy was related to eGFR, urinary protein levels, and the incidence of hypertension before the treatment. The side effects of administration of CsA alone were significantly lower than those of the combination treatment.

Key words: Elderly idiopathic membranous nephropathy; Low doses; Glucocorticoids; Cyclosporine A

\section{INTRODUCTION}

Membranous nephropathy is the most common pathological type of nephropathy in elderly patients. In addition to the massive proteinuria and hypoalbuminemia, the clinical complications of membranous nephropathy include hypertension, renal failure, hyperlipidemia, infections, hypercoagulation, and thromboembolism (Cattran et al., 2001; Cattran et al., 2007; Ronco and Debiec, 2011). Therefore, our primary objectives were to reduce proteinuria, increase the plasma albumin levels, and improve the symptoms of nephrotic syndrome (NS); moreover, we aimed to decrease the complications associated with membranous nephropathy (Yoon et al., 2011; Brown et al., 2012; Verde et al., 2012). Currently, the treatment of membranous nephropathy is mainly based on the degree of proteinuria; the treatment includes administration of angiotensin-converting enzyme inhibitors (ACEIs) or angiotensin receptor blockers (ARBs) (Cattran, 2005; Lai, 2007; Waldman and Austin, 2012) and administration of a combination of hormones and immunosuppressive therapy. There are different views about the use of immunosuppressive therapy, and some studies suggest that cytotoxic drugs could decrease the proteinuria and improve the clinical remission rate in patients with membranous nephropathy. Recently, some studies showed that administration of the calcineurin inhibitor cyclosporine A (CsA) at conventional doses $\left(3-5 \mathrm{mg} \cdot \mathrm{kg}^{-1} \cdot \mathrm{day}^{-1}\right)$ significantly improved the prognosis of NS patients with worsened renal functions but was associated with complications such as hepatonephric dysfunction and infections (Goumenos et al., 2007; Ponticelli and Passerini, 2010). Further, the administration of CsA alone for the treatment of membranous nephropathy explored previously was partially effective. However, whether the administration of low-dose CsA (1-3 $\left.\mathrm{mg} \cdot \mathrm{kg}^{-1} \cdot \mathrm{day}^{-1}\right)$ alone will be effective in a special population such as the elderly patients and the side effects of this treatment have not been reported thus far. Here, we have reported the effects of administration of low-dose CsA alone or in combination with a low-dose hormone therapy in elderly patients with membranous nephropathy.

\section{MATERIAL AND METHODS}

\section{General information}

We reported the cases of 27 elderly patients ( 15 men and 12 women; age, 65-81 years) with idiopathic membranous nephropathy who were admitted to our hospital between January 2008 and January 2013. Their condition was confirmed by biopsy, and the patients were followed up for 6 months or more. The treatment duration was 6 months to 2 years; mean duration, $13.5 \pm 6.2$ months. The follow-up period was 6-48 months. The renal pathological staging was stage I and II. Patients with autoimmune diseases, cancer, infections, and drugs were excluded, and the follow-up time was not less than 6 months. This study was conducted in ac- 
cordance with the declaration of Helsinki. This study was approved by the Ethics Committee of the Navy General Hospital of PLA. Written informed consent was obtained from all participants.

\section{Research methods}

Our study was a prospective randomized controlled study of administration of lowdose CsA alone or in combination with a low-dose hormone therapy in elderly patients with idiopathic membranous nephropathy.

\section{Inclusion criteria}

The renal tissue specimens of all patients were examined using light microscopy, immunofluorescence, and electron microscopy, and the condition of the patients was pathologically diagnosed as idiopathic membranous nephropathy stage I and II. The 24-h protein levels were $>4 \mathrm{~g}$. The patients were older than 65 years, and the treatment duration was not less than six months, and the follow-up time was not less than six months.

\section{Exclusion criteria}

1) Secondary membranous nephropathy induced by secondary factors such as autoimmune diseases, cancer, infections and drugs, or atypical membranous nephropathy. 2) HIV infection. 3) Patients diagnosed with malignant tumor infection. 4) Patients with active hepatitis $\mathrm{B}$ or $\mathrm{C}$ or with positive replication indexes.

\section{Grouping}

The patients were divided into the following two groups according to their different treatment options: 1) group A: patients receiving low-dose CsA alone and 2) group B: patients receiving a combination of low-dose glucocorticoids (methylprednisolone) and low-dose CsA. The initial dose of the methylprednisolone tablet was $0.4 \mathrm{mg} \cdot \mathrm{kg}^{-1} \cdot \mathrm{day}^{-1}$, which gradually decreased after 8 to 12 weeks administration; the total duration of treatment was 6-12 months. The initial dose of CsA was $2 \mathrm{mg} \cdot \mathrm{kg}^{-1} \cdot$ day $^{-1}$, and the treatment duration was not less than six months. The plasma CsA concentration was monitored, so that it could be maintained at 80 $120 \mathrm{ng} / \mathrm{mL}$. In patients with plasma CsA concentration different from the standard, the dose was gradually increased or decreased by $25 \mathrm{mg}$ each time; the total duration of treatment was 6-12 months. If the serum creatinine ratio increased by $30 \%$ of the baseline value after treatment, the dose of CsA was decreased daily by $25 \mathrm{mg}$. We used the Xinshandiming tablet (Novartis Pharma $\mathrm{GmbH}$, Germany) containing $25 \mathrm{mg}$ CsA/tablet. Meizhuole (Pfizer Italia Srl, Italy) containing $4 \mathrm{mg}$ methylprednisolone/tablet was used as the methylprednisolone tablet.

\section{Eluting treatment}

The patients admitted were required to maintain their serum creatinine (Scr) levels $\leq 178 \mu \mathrm{M}$, and thus, they were administered a single-dose of the ARB-class drug valsartan capsule $80 \mathrm{mg}$ (Novartis Pharma Co., Ltd., Beijing). All the patients were administered lipidlowering drugs and anti-platelet adhesion drugs according to their specific conditions, and the blood pressure was controlled $<140 / 90 \mathrm{mmHg}$. 


\section{Exit criteria}

The exit criteria were serious infections; worsening of the renal functions; increase in the Scr levels by $50 \%$ of the baseline values, which failed to recover after the reduction in the dose of medication; steroid glycosuria poorly controlled by the medication; and severe gastrointestinal bleeding.

\section{Observation indexes}

Clinical indexes such as monthly levels of Scr, serum albumin, and 24-h urinary protein were observed before and after the treatment. For examination of hematuria, the patient emptied the bladder at 7 a.m. in the morning, and then, maintained a 24-h record of the urine and accurately recorded the volume to test the 24-h urinary protein levels. We used the Siemens ADVIA 2400 biochemical analyzer, and the urinary protein levels were determined using immunonephelometry, and the serum and urine creatinine levels were detected using the enzyme method.

The modification of diet in renal disease formula and the chronic kidney disease epidemiology collaboration was used to calculate the estimated glomerular filtration ratio (eGFR).

\section{Criteria of clinical efficacy}

After 6-12 months of treatment, we determined the effectiveness and remission rate. Complete remission (CR) was defined as urinary protein level $\leq 0.3 \mathrm{~g} /$ day, serum albumin level $>35 \mathrm{~g} / \mathrm{L}$, stable renal function (increase in the Scr level was less than $15 \%$ of the baseline value); partial remission (PR) was defined as the decrease in urinary protein level by more than $50 \%$ of the baseline value, urinary protein level $\leq 3.5 \mathrm{~g} /$ day, stable renal function; and no remission: the decrease in the urinary protein level was $50 \%$ of the baseline value, or the urinary protein level was $>3.5 \mathrm{~g} /$ day, or the Scr level was more than $50 \%$ of the baseline value. The clinical remission rate $=(\mathrm{CR}+\mathrm{PR}) /$ total number of patients $\times 100 \%$.

Safety: 1) the adverse reactions observed during the treatment were infection, osteonecrosis, steroid glycosuria, and hepatonephric toxicity, and patients discontinued treatment because of the above factors. 2) The recurrence rate after drug withdrawing.

\section{Statistical analysis}

The SPSS13.0 software was used for the statistical analysis. The measurement data are reported as means $\pm \mathrm{SD}$. Intergroup comparison was performed using the independent sample $t$ test; the enumeration data were analyzed using chi-square test, with $\mathrm{P}<0.05$ considered to be the statistically significant difference.

\section{RESULTS}

\section{General information}

No statistically significant difference was observed in gender, age, pre-treatment Scr levels, eGFR, and 24-h urinary protein levels between the 2 groups $(\mathrm{P}>0.05$; Table 1$)$. 
Table 1. Comparison of general information of the 2 groups (means $\pm \mathrm{SD}$ ).

\begin{tabular}{lclccc}
\hline Group $(\mathrm{N})$ & Age & Gender & Urinary protein quantitation $(\mathrm{g} /$ day) & eGFR (mL/min) & Pre-treatment serum creatinine \\
\hline A (14) & $75.1 \pm 8.2$ & 10 males and 4 females & $7.2 \pm 3.4$ & $69.6 \pm 10.3$ & $98.8 \pm 15.1$ \\
B (13) & $74.8 \pm 7.9$ & 10 males and 3 females & $7.5 \pm 3.8$ & $70.9 \pm 11.9$ & $91.6 \pm 20.9$ \\
\hline
\end{tabular}

\section{Comparison of efficacy}

No statistically significant difference was observed in the CR and PR rates of groups $\mathrm{A}$ and $\mathrm{B}$; the overall remission rate in group $\mathrm{A}$ was $69.2 \%$, which was slightly higher than that in group B (Table 2).

Table 2. Efficacy comparison of the two groups (means $\pm \mathrm{SD}$ ).

\begin{tabular}{lccc}
\hline Group $(\mathrm{N})$ & CP cases $(\%)$ & PR cases $(\%)$ & Overall remission case (\%) \\
\hline A $(14)$ & $1(7.1)$ & $7(50.0)$ & $8(57.1)$ \\
B $(13)$ & $2(15.4)$ & $6(46.2)$ & $8(61.6)$ \\
\hline
\end{tabular}

\section{Comparison of complications}

One patient in group A showed a combination of severe pneumonia and immune hypofunction after 3 months (CD4 < 200), and thus discontinued treatment; 1 patient had hypertension; 3 patients showed a $>50 \%$ increase in the Scr levels within 2 weeks of treatment, and thus, the CsA dose was decreased. The Scr levels of 2 of these 3 patients recovered, but the renal function of 1 patient did not stabilize, and thus, this patient discontinued treatment. In group B, 3 patients showed steroid diabetes; of these, 2 were treated with insulin, and 1 patient discontinued treatment because of the difficulty in controlling the blood sugar levels. Two patients showed severe pneumonia after 2 months, and 1 patient showed simultaneous recurrent urinary tract infection and immune hypofunction (CD4 < 200), and thus discontinued the treatment; 1 patient showed hypertension; the pre-existing hypertension in the case of 1 patient became difficult to control, and thus, this patient received antihypertensive medications. Four patients showed a $>50 \%$ increase in serum creatinine levels within 2 weeks of treatment. The CsA dose was decreased in these patients; subsequently, the Scr levels of 2 patients recovered, while 2 patients discontinued treatment because the renal function did not recover. The rates of complications were significantly higher in group B than in group A (Table 3).

\begin{tabular}{|c|c|c|c|c|c|c|}
\hline Group $(\mathrm{N})$ & $\begin{array}{c}\text { Steroid } \\
\text { diabetes }(\%)\end{array}$ & $\begin{array}{l}\text { Hypertension or the blood } \\
\text { pressure was difficult to } \\
\text { be controlled }(\%)\end{array}$ & Infection (\%) & $\begin{array}{l}\text { Serum creatinine } \\
\text { increasing }>50 \%\end{array}$ & Quit (\%) & $\begin{array}{c}\text { Cases of severe } \\
\text { complications }(\%)\end{array}$ \\
\hline A(14) & $0(0)$ & $1(7.1)$ & $1(7.1)$ & $3(21.4)$ & $2(14.3)$ & $5(35.7)$ \\
\hline B (13) & $3(23.1)$ & $2(15.4)$ & $2(15.4)$ & $4(30.8)$ & $4(30.8)$ & $11(84.6)^{*}$ \\
\hline $\mathrm{t}$ & 0.098 & & & & 0.385 & 0.018 \\
\hline
\end{tabular}

*Compared with the A group, $\mathrm{P}<0.05$. 


\section{Comparison of clinical data}

Examination of the pretreatment clinical data of patients who achieved remission and those who did not showed that after the treatment, the rates of hypertension and 24-h urinary protein levels were significantly lower in patients who achieved remission than in those who did not achieve remission; although the pre-treatment eGFR of patients who achieved remission was significantly higher than that of patients who did not achieve remission, no significant difference was observed in gender, age, and pathological stage (I, II proportional) between patients who achieved remission and those who did not (Table 4).

Table 4. Clinical data comparison between the remission and non-remission patients (means $\pm \mathrm{SD}$ ).

\begin{tabular}{lcccccc}
\hline Group (N) & $\begin{array}{c}\text { Gender } \\
\text { (male/female) }\end{array}$ & Age (years) & $\begin{array}{c}\text { Hypertension } \\
\text { cases (\%) }\end{array}$ & $\begin{array}{c}\text { Urinary protein } \\
\text { excretion (g/day) }\end{array}$ & $\begin{array}{c}\text { Pathological } \\
\text { stage (I/II) }\end{array}$ & $\begin{array}{c}\text { Pre-treatment } \\
\text { eGFR (mL/min) }\end{array}$ \\
\hline Remission (16) & $10 / 6$ & $71.6 \pm 9.1$ & 8 cases (50.0) & $6.5 \pm 3.1$ & $12 / 4$ & $70.1 \pm 12.7$ \\
Non- Remission (11) & $10 / 1$ & $75.9 \pm 8.7$ & 10 cases (90.9)* & $9.8 \pm 4.6^{*}$ & $5 / 6$ & $60.2 \pm 10.4^{*}$ \\
$\mathrm{t}$ & 0.183 & 0.231 & 0.042 & 0.035 & 0.224 & 0.043 \\
\hline
\end{tabular}

*Compared with the Remission, $\mathrm{P}<0.05$.

\section{DISCUSSION}

Idiopathic membranous nephropathy accounts for approximately $10 \%$ of primary glomerular diseases in China; the ratio of male to female incidence of idiopathic membranous nephropathy is $2: 1$. This disease is more common among the elderly, and the pathological characteristic of idiopathic membranous nephropathy is the diffuse thickening of the capillary basement membrane caused by deposition of the immune complex under the glomerular epithelial cells. Studies conducted in China and abroad have shown similar results about the pathological features of NS in elderly patients. The condition of approximately $50 \%$ of the patients with idiopathic membranous nephropathy and NS naturally progresses toward renal failure; however, in some patients, active treatment alleviates the proteinuria, and thus, improves the renal survival rate (Yoon et al., 2011; Brown et al., 2012; Verde et al., 2012).

Cattran (2005) proposed that the patients with idiopathic membranous nephropathy should be treated according to the risk stratification. Low-risk patients (with normal Scr levels, urinary protein level $<4 \mathrm{~g}$ /day for 6 months, and low risk of disease progression) should only be administered ACEIs, ARBs, anticoagulants, and treatments for regulating lipid levels and controlling blood pressure and other symptoms, and their urinary protein levels and renal function should be periodically monitored. Moderate-risk patients (with normal or near normal levels of Scr and stable urinary protein levels between 4 and $8 \mathrm{~g} /$ day for 6 months) should be symptomatically treated and observed for 6 months. During this period, if the renal function is aggravated and cannot be relieved, then the patients should be treated with immunosuppressants [glucocorticoids combined with chlorambucil or cyclophosphamide (CTX)]. High-risk patients (with abnormal or worsened renal function and urinary protein levels $>8 \mathrm{~g}$ /day for 6 months) should be administered a combination therapy of hormones and cytotoxic drugs on the basis of low-risk and middle-risk therapies. If the treatment fails and the renal function worsens, CsA, mycophenolate mofetil (MMF), tacrolimus (FK506), and other new immunosuppressive drugs should be administered. The above treatment should not be administered 
to patients with Scr levels $>352 \mu \mathrm{M}$, diffuse glomerulosclerosis, or extensive renal interstitial fibrosis (Cattran, 2005; Lai, 2007; Waldman and Austin, 2012).

A previous study suggests that the first-line treatment for membranous nephropathy is a combination of glucocorticoids and chlorambucil or CTX, but the incidences of adverse reactions have limited the use of this treatment (Cattran et al., 2007). A comparison of the outcomes of combination therapy using different immunosuppressants showed no significant difference in the overall remission rates among the CTX, MMF, and CsA groups ( $P>0.05)$, and the remission rate was the highest in patients receiving a combination of prednisone and CsA. Renal function before the treatment is an important prognostic factor (Goumenos et al., 2007; Ponticelli and Passerini, 2010).

A single-blind, randomized, controlled trial was performed at 11 kidney disease centers of North America, and this study evaluated the use of CsA in patients with steroid-resistant idiopathic membranous nephropathy. The results of this study showed that administration of low-dose CsA was safe and effective, but the authors also pointed out that CsA was expensive, and the rate of recurrence of membranous nephropathy was high after drug withdrawal, and some patients developed adverse reactions, including nephrotoxicity and long-term renal interstitial fibrosis. Goumenos et al. (2007) compared the effects of concomitant administration of hormones with CsA and cytotoxic drugs in treating patients with idiopathic membranous nephropathy. The results showed that compared to cytotoxic drugs, CsA had a higher remission rate (85 vs 55\%) and fewer adverse reactions, but had a higher relapse after drug withdrawal and frequent serious adverse reactions (10 vs 4\%). Therefore, Cattran et al. (2001) explored the application of CsA treatment in membranous nephropathy patients and recommended a low dose of 3-4 mg $\mathrm{kg}^{-1} \cdot \mathrm{day}^{-1}$ for at least 6 months. Meanwhile, Ponticelli and Passerini (2010) recommended a dose of $1-3 \mathrm{mg} \cdot \mathrm{kg}^{-1} \cdot \mathrm{day}^{-1}$ of CsA for more than 2 years to prevent recurrence. A small dose usually does not cause hypertension, and the renal insufficiency associated with this condition was resolved when the dosage was reduced. A low dose of CsA relieved proteinuria and effectively protected the renal function in several patients with idiopathic membranous nephropathy. Although adverse reactions were common and predictable, the criteria of regularity and quantitation, as well as therapeutic drug monitoring, significantly decrease the risk of renal toxicity (Cattran et al., 2007).

Majority of researchers have recommended the combined therapy of glucocorticoids and cytotoxic drugs for the treatment of idiopathic membranous nephropathy. These recommendations were made on the basis of studies performed in adults, and much information has not been reported about treatment of elderly patients with idiopathic membranous nephropathy. Active treatment should be sought for the remission of membranous nephropathy in elderly patients taking into account the characteristics of elderly people and long-term side effects of hormone therapy. Administration of hormones and immunosuppressive therapy to elderly patients continues to be controversial. The results of a large number of evidence-based medical studies showed that despite short-term or long-term oral administration, administration of glucocorticoids alone showed no significant benefit in the induction of clinical remission and renal protection in patients with membranous nephropathy. Schieppati et al. (2004) systematically evaluated the effect of administration of immunosuppressants in 1025 patients with idiopathic membranous nephropathy and NS, and they found that administration of glucocorticoids alone could improve neither the $\mathrm{CR}$ and PR rates of proteinuria nor the long-term survival of the kidney.

A large-dose combination of hormones and immunosuppressants showed higher side 
effects in elderly patients, because the rate of metabolism of glucocorticoids is negatively correlated with age. The clearance rate in the elderly reduced to one-third of that in the younger population, and the elderly patients were much more likely to develop infections, hypertension, steroid diabetes, osteoporosis, and other complications, including life-threatening events. Therefore, the usage glucocorticoids is not advocated. A small-dose long-term hormone therapy would be favorable in the prognosis of elderly patients with higher life expectancy and uncertain estimated effects. To date, few randomized controlled studies with immunosuppressant therapy alone have been performed in patients with idiopathic membranous nephropathy and NS. Although the combined therapy of glucocorticoids has proven effective in relieving proteinuria and protecting renal function (grade A in evidence-based medicine), this therapy continues to have serious side effects in the treatment of elderly patients with membranous nephropathy. CTX, MMF, and tripterygium glycosides induce side effects such as vascular inflammation, severe gastrointestinal reactions, bone marrow suppression, infection, and hemorrhagic cystitis, but exhibit no effects in a considerable portion of elderly patients. Therefore, they should not be used as conventional medication for the treatment of elderly patients with membranous nephropathy. A recent study has reported the use of rituximab and a phospholipase $\mathrm{A} 2$ receptor antagonist, but the clinical of this therapy require further investigation, and therefore, this treatment cannot be recognized as first-line medication for the treatment of idiopathic membranous nephropathy (Schieppati et al., 2004; Bruschi et al., 2011; Cravedi et al., 2011; Hoxha et al., 2011; Qin et al., 2011).

Some studies have shown that even a small dose of CsA significantly improves proteinuria and aggravation of renal function in patients with idiopathic membranous nephropathy (Jha et al., 2007; Couser, 2012). However, among the special population such as elderly patients with idiopathic membranous nephropathy, the efficacy and side effects of administration of low-dose CsA alones have not been reported thus far. In this study, we administered low-dose CsA alone or in combination with a low-dose hormone to treat elderly patients with idiopathic membranous nephropathy.

Our results showed that although no significant difference was observed in the CR and $\mathrm{PR}$ of proteinuria between groups $\mathrm{A}$ and $\mathrm{B}$, the overall remission rate in the group $\mathrm{B}$ was slightly higher than that in the group A. All patients achieved a remission rate of about $60 \%$. Compared to patients in group A, those in groups B showed significant differences in the rates of discontinuation and morbidity, which indicated that even a small dose of hormone had some side effects. Analysis of relevant factors of remission rate showed no obvious relationship between the remission rate and gender, age, and membranous nephropathy stage I or II. However, remission was associated with hypertension ratio, 24-h urinary protein excretion, and pre-treatment renal functions, such as pre-treatment combined hypertension, severe urinary protein excretion, and renal dysfunction. These results were similar to those reported previously (Branten et al., 2007).

In summary, we found that administration of low-dose CsA alone or in combination with a low-dose hormone was effective in the treatment of elderly patients with idiopathic membranous nephropathy, and this treatment significantly improved the prognosis of patients with NS and renal function aggravation. In particular, administration of low-dose CsA alone was not only effective but it also showed fewer side effects than those observed in combination with hormone therapy; further, low-dose CsA is safe for use in the clinic. The number of patients in our study was less, and further studies with a large sample size and longer followup period should be performed in the future. 


\title{
Conflicts of interest
}

\author{
The authors declare no conflict of interests.
}

\section{REFERENCES}

Branten AJ, du Buf-Vereijken PW, Vervloet M and Wetzels JF (2007). Mycophenolate mofetil in idiopathic membranous nephropathy: a clinical trial with comparison to a historic control group treated with cyclophosphamide. Am. $J$. Kidney Dis. 50: 248-256.

Brown CM, Scheven L, O'Kelly P, Dorman AM, et al. (2012). Renal histology in the elderly: indications and outcomes. J. Nephrol. 25: 240-244.

Bruschi M, Carnevali ML, Murtas C, Candiano G, et al. (2011). Direct characterization of target podocyte antigens and auto-antibodies in human membranous glomerulonephritis: Alfa-enolase and borderline antigens. J. Proteomics 74: 2008-2017.

Cattran D (2005). Management of membranous nephropathy: when and what for treatment. J. Am. Soc. Nephrol. 16: 1188-1194.

Cattran DC, Appel GB, Hebert LA, Hunsicker LG, et al. (2001). Cyclosporine in patients with steroid-resistant membranous nephropathy: a randomized trial. Kidney Int. 59: 1484-1490.

Cattran DC, Alexopoulos E, Heering P, Hoyer PF, et al. (2007). Cyclosporin in idiopathic glomerular disease associated with the nephrotic syndrome: workshop recommendations. Kidney Int. 72: 1429-1447.

Couser WG. (2012). Basic and translational concepts of immune-mediated glomerular diseases. J. Am. Soc. Nephrol. 23 : 381-399.

Cravedi P, Sghirlanzoni MC, Marasà M, Salerno A, et al. (2011). Efficacy and safety of rituximab second-line therapy for membranous nephropathy: a prospective, matched-cohort study. Am. J. Nephrol. 33: 461-468.

Goumenos DS, Katopodis KP, Passadakis P, Vardaki E, et al. (2007). Corticosteroids and cyclosporine A in idiopathic membranous nephropathy: higher remission rates of nephrotic syndrome and less adverse reactions than after traditional treatment with cytotoxic drugs. Am. J. Nephrol. 27: 226-231.

Hoxha E, Harendza S, Zahner G, Panzer U, et al. (2011). An immunofluorescence test for phospholipase- $\mathrm{A}_{2}$-receptor antibodies and its clinical usefulness in patients with membranous glomerulonephritis. Nephrol. Dial. Transplant. 26: $2526-2532$.

Jha V, Ganguli A, Saha TK, Kohli HS, et al. (2007). A randomized, controlled trial of steroids and cyclophosphamide in adults with nephrotic syndrome caused by idiopathic membranous nephropathy. J. Am. Soc. Nephrol.18: 1899-1904.

Lai KN (2007). Membranous nephropathy: when and how to treat. Kidney Int. 71: 841-843.

Ponticelli C and Passerini P (2010). Management of idiopathic membranous nephropathy. Expert Opin. Pharmacother. 11: 2163-2175.

Qin W, Beck LH Jr, Zeng C, Chen Z, et al. (2011). Anti-phospholipase A2 receptor antibody inmembranous nephropathy. J. Am. Soc. Nephrol. 22: 1137-1143.

Ronco P and Debiec H (2011). Antigen identification in membranous nephropathy moves toward targeted monitoring and new therapy. J. Am. Soc. Nephrol. 21: 564-569.

Schieppati A, Perna A, Zamora J, Giuliano GA, et al. (2004). Immunosuppressive treatment for idiopathic membranous nephropathy in adults with nephrotic syndrome. Cochrane Database Syst. Rev. 18: CD004293.

Verde E, Quiroga B, Rivera F and Lopez-Gomez JM (2012). Renal biopsy in very elderly patients: data from the Spanish Registry of Glomerulonephritis. Am. J. Nephrol. 35: 230-237.

Waldman M and Austin HA 3rd (2012). Treatment of idiopathic membranous nephropathy. J. Am. Soc. Nephrol. 23: 1617-1630.

Yoon HE, Shin MJ, Kim YS, Choi BS, et al. (2011). Clinical impact of renal biopsy on outcomes in elderly patients with nephrotic syndrome. Nephron. Clin. Pract. 117: c20-c27. 